\title{
EDITORIAL
}

\section{A message for 2017}

\author{
Journal of Human Genetics (2017) 62, 517-519; doi:10.1038/jhg.2017.29
}

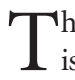
he third year as Editor-in-Chief for the Journal of Human Genetics is over. The Impact Factor has remained at a similar level (2.487). The Journal of Human Genetics published 158 papers in 2016. We prepared a special issue describing non-coding RNAs in human diseases in 2016, which was published in January 2017. I would like to express my appreciation to guest editors, Drs Naohiko Seki and Akira Hata, and all the contributors for their hard work. I hope the special issue will attract attention from a broad readership.

In 2016, we celebrated Professor Yoshinori Ohsumi receiving 2016's Nobel Laureate in Physiology or Medicine. His outstanding achievements in autophagy have opened up new research fields related to human diseases. I myself discovered disease-causing mutations of WDR45 encoding WIPI4, a core protein in autophagy. I expect more manuscripts related to autophagy in the Journal of Human Genetics.

Alice Abdel Aleem
David Adams
Sarita Agarwal
JM Akey
Yoko Aoki
Zohar Argov
Takuro Arimura
Antonio Arnaiz-Villena
Rafael Artuch
Tomoyuki Aruga
Danillo G Augusto
Hiroyuki Awano
Anitha Ayyappan Pillai
Masaya Baba
Seiamak Bahram
Shabeesh Balan
Shanti Balasubramaniam
Roland Baron
Chiara Batini
Claire Bellis
Minako Beppu
Stephane Bezieau
Rui Bi
Gerhard Binder
Alan Bittles
Susan H Blanton
Nenad Blau

Martin Bodner
Luisa Bonafe
Jacquelyn Bond
Gisele Bonne
Chehrazade Brick
Natasha J Brown
Cristian Capelli
Luis Carvajal-Carmona
Denise Cavalcanti
Jeffrey S Chamberlain
Takeshi Chiyomaru
Murim Choi
Brian Hon-Yin Chung
Angus Clarke
Mark Corbett
Timothy M Cox
Fulvio Cruciani
Veronika Csakyova
Goran Cuturilo
Yehia Daaka
Amber Dahlin
Makoto Daimon
Diodato Daria
Mehul Dattani
Andrew Dauber
Peter De Knijff
Danny N Dhanasekaran

The sister online open-access journal, Human Genome Variation, was registered in PubMed in 2016. I feel Human Genome Variation is steadily progressing.

In 2017, we are again planning a special issue. I am looking forward to it, and of course to receiving more exciting manuscripts for the Journal of Human Genetics from all over the world.

\section{Naomichi Matsumoto Editor-in-Chief Journal of Human Genetics}

\section{ACKNOWLEDGEMENTS}

The Editor-in-Chief, on behalf of the Journal of Human Genetics Editorial Board and the Japan Society of Human Genetics, gratefully acknowledges the generous support from peer reviewers in carefully assessing manuscripts under consideration. The following individuals provided reviews of manuscripts submitted to the Journal of Human Genetics in 2016:

Kimon Divaris

Yolanda Martin Santo Domingo

Nataliya Di Donato

Rajkumar Dorajoo

Ana T Duggan

Yusuke Ebana

Simon Edvardson

Thore Egeland

Thomas Eggermann

Alison Elliott

Akihiro Fujimoto

Ken-ichi Fujita

Maki Fukami

Hiroshi Furukawa

Brenda Gerull

Lina Ghaloul-Gonzalez

Wendy A Gold

Atsushi Goto

Shin Goto

Yusuke Goto

Tsuyoshi Hachiya

Nobuhiko Haga

Koichi Hagiwara

Kazuo Hara

Naoki Harada

Tomonobu Hasegawa

Nobuhiro Hata
Takaaki Hayashi

Takeharu Hayashi

Yukiko K Hayashi

Madhuri Hegde

Deborah Himes

Naomi Hino-Fukuyo

Makito Hirano

Akira Hirasawa

Tomomitsu Hirota

Yuki Hitomi

Michi Hofreiter

Yukio Horikawa

Paul Horton

Kazuyoshi Hosomichi

Yoshihiro Hotta

David A Hughes

Martijn Huynen

Vivian Hwa

Shingo Ichimiya

Masashi Ikeda

Minako Imamura

Issei Imoto

Toshifumi Inada

Hidetoshi Inoko

Ituro Inoue

Jun Inoue

Satoshi Inoue 
Melita Irving

Takayuki Ishige

Naoko Ishihara

Atsushi Ishii

Kinya Ishikawa

Hiroyuki Ishiura

Minoru Isomura

Kaoru Ito

Atsushi Iwata

Akira Iyoda

Jessie Jacobsen

Zandra Jenkins

Zi-Bing Jin

Mark Jobling

Alexander A Jorge

Eric Jorgenson

Vera M Kalscheuer

Naoyuki Kamatani

Yoichiro Kamatani

Tadashi Kaname

Piranit N Kantaputra

Naoyuki Kataoka

Mitsuhiro Kato

Aya Kawasaki

Haig H Kazazian

Jennifer Kearney

Christine Keyser

Atsuo Kikuchi

Takashi Kikuiri

Ryosuke Kimura

Akira Kinoshita

Takashi Kinoshita

Shin-ichiro Kitajiri

Kazuma Kiyotani

Eva Klopocki

Anita Kloss-Brandstatter

Kazuhiro Kobayashi

Masahisa Kobayashi

Takashi Kobayashi

Yuta Kochi

Tomomi Kogiso

Hiroyuki Kondo

Qing-Peng Kong

Uwe Kornak

Yoshimasa Kosaka

Tomoki Kosho

Motomichi Kosuga

Mitsuru Kubota

Tomu Kuchikata

Akihiro Kume

Hiroki Kurahashi

Kenji Kurosawa

Alena Kushniarevich

Kazuyoshi Kuwano

Chris Lau

Ekkehart Lausch

Hwan Young Lee

Ming-Ta Michael Lee

Tomoko Lee

Chiara Leoni

Gaetan Lesca
Hui Li

Jingmei Li

Yu-Chun Li

Zhaohai Li

Shih-Hua Lin

Shili Lin

Raymond Louie

Siew-Kee Low

Friedrich C Luft

Artem Lysenko

Deborah JG Mackay

Shiro Maeda

Surakameth Mahasirimongkol

Assumpcio Malgosa

Joan Marini

Saber Masmoudi

Muneyuki Masuda

Keiko Matsubara

Tatsuo Matsunaga

Mari Matsuo

Masafumi Matsuo

Ryosuke Matsushita

Giovanni Meola

Laurence Michel-Calemard

Toshimi Michigami

Ohsuke Migita

Tohru Minamino

Kazuharu Misawa

Hiroyuki Mishima

Anna Mitchell

Fuyuki Miya

Maiko Miyagawa

Taku Miyagawa

Noriko Miyake

Toshinobu Miyamoto

Pierre Moffatt

Kaoru Mogushi

Michal Mokry

Yukihide Momozawa

Darren Monckton

Toshiaki Monkawa

Lluis Montoliu

Takeshi Morihara

Hiroyuki Morita

Amelia Morrone

Hideaki Moteki

Imen Moumni

Stefan Mundlos

Masaaki Muramatsu

Jun Murotsuki

Taisei Mushiroda

Kaori Muto

Keiko Myouzen

Takeshi Nagamatsu

Masao Nagasaki

Masanori Nakagawa

Naoki Nakagawa

Hiroshi Nakajima

Kimitoshi Nakamura

Eiji Nanba

Aya Narita
Muhammad Imran Naseer

Sergey Nejentsev

Isabelle Nelson

Gael Nicolas

Fumichika Nishimura

Gen Nishimura

Jo Nishino

Hisahide Nishio

Emiko Noguchi

Nijiro Nohata

Hisashi Noma

Hironao Numabe

Katsuhisa Ogata

Tsutomu Ogata

Tomoo Ogi

Hiromi Ogura

Hirofumi Ohashi

Jun Ohashi

Toya Ohashi

Koichiro Ohmura

Seiko Ohno

Yutaka Ohsawa

Tohru Ohta

Akira Ohtake

Akira Oka

Yukinori Okada

Nobuhiko Okamoto

Tatsushi Okayama

Yasushi Okazaki

Akihisa Okumura

Torayuki Okuyama

Morten Salling Olesen

Michelle L Olsen

Yosuke Omae

Osamu Onodera

Yoshihiro Onouchi

Raffaella Origa

Hisao Osada

Ichiro Ota

Masao Ota

Takeshi Otowa

Jurg Ott

Kouichi Ozaki

Takeshi Ozeki

Catherine Park

Martin Peifer

Min-Sheng Peng

Dorota Piekutowska-Abramczuk

Manop Pithukpakorn

Huntington Potter

Markus Preising

Dariusz Pytel

Xuebin Qi

Huaizhen Qin

Chisei Ra

Gudrun Rappold

Frank Rauch

Gloria Ribas

Martin B Richards

M Rienstra

Carlo Rivolta
Lutz Roewer

Heiko Ruetter

Deborah Rund

Kentaro Sahashi

Shinji Saito

Shinji Saito

Yoshiaki Saito

Shinji Saitoh

Hirotomo Saitsu

Norio Sakai

Akihiro Sakurai

Antonio Salas

Osamu Samura

Aiko Sasaki

Suguru Sato

Marco Savarese

Naohiko Seki

Masayuki Sekine

Jun Sese

Iman Shaheen

Hong Shi

Atsushi Shibata

Daichi Shigemizu

Takashi Shiina

Haruo Shimazaki

Kenji Shimizu

Masaru Shimura

Masaki Shiota

Yuichi Shiraishi

David Sillence

Isabel Silveira

Malte Spielmann

Hariharan Sreedharan

Hideo Sugie

Chang Sun

Fei Sun

Carolyn K Suzuki

Yoichi Suzuki

Hayato Tada

Atsushi Tajima

Go Tajima

Atsushi Takahashi

Yuji Takahashi

Kyoko Takano

Shigetsugu Takano

Miho Takaoka

Hiroshi Takashima

Toshiki Takenouchi

Yasuhiro Takeshima

Fumihiko Takeuchi

Takashi Taki

Mayumi Tamari

Hannah Tamary

Tiong Y Tan

Masashi Tanaka

Mariko Taniguchi-Ikeda

Chizu Tanikawa

Kousuke Tanimoto

Marco Tartaglia

Chikashi Terao

Shunji Tomatsu 
Kazuhito Tomizawa

Pamela Tran

Wieslaw Trzeciak

Shoji Tsuji

Chris Tyler-Smith

Tiina Tyni

Henna Tyynismaa

Andreas Tzschach

Hiroaki Uchida

Mitsuharu Ueda

Takehiro Ueda

Shin-ichi Usami

TJ Visser
Keiko Wakui

Tom Walsh

Chaolong Wang

Fan Wang

Jingmin Wang

Hansi Weissensteiner

Susan White

Stephen D Wilton

Rongling $\mathrm{Wu}$

Ichiro Yabe

Ryo Yamada

Takeshi Yamada

Takanori Yamagata
Hiroyuki Yamagishi

Seiji Yamaguchi

Kazuhiro Yamakawa

Toshiyuki Yamamoto

Keiko Yamazaki

Yao Yang

Yong Yang

Zhaoqing Yang

Takahiro Yasui

Michio Yasunami

Long Yi

Nengjun Yi

Toshifumi Yokota
Kunihiro Yoshida

Hiroshi Yoshihashi

Hirofumi Yoshino

Koh-ichiro Yoshiura

Massimo Zeviani

Fangyuan Zhang

Xiao-Ming Zhang

Pingping Zheng

Hui Zhou

Asima Zia 